brachte Tatsachen sind in diesem Verfahren jedoch grundsätzlich unbeachtlich (vgl. \$137 Abs. 2 VwGO).

[33] Die Feststellung des Oberverwaltungsgerichts, die Einbeziehung von Waldflächen in einem Umfang von rund 250 ha sei aus Gründen der Vermeidung unternehmensbedingter landeskultureller Nachteile im Trassenbereich und einer durchgängigen eigentumsrechtlichen Neuordnung gerechtfertigt, wird durch das Revisionsvorbringen nicht schlüssig in Frage gestellt. Allein der Umfang des Flurbereinigungsgebiets oder dessen Relation zur Bedarfsfläche begründet keine Verletzung von Bundesrecht. Dass das Ziel der Vermeidung unternehmensbedingter landeskultureller Nachteile die Einbeziehung von Waldflächen nicht oder nicht in dieser Größenordnung erfordert oder nicht im Vordergrund der Maßnahme steht, ist nicht erkennbar. ..

DOI: 10.1007/s10357-010-1853-5

\section{Die Entwässerung eines Biotops ist dann unzulässig, wenn das Biotop nicht erst durch Bewirtschaftungsbeschränkung entstanden ist}

\section{SächsNatschG §§26, 41; VwGO §80 Abs. 5}

1. Nach $\$ 26$ Abs. 2 SächsNatSchG sind alle Handlungen, die zu einer Zerstörung oder zu sonstigen erheblichen oder nachhaltigen Beeinträchtigungen der besonders geschützten Biotope führen können, verboten.

2. Der Eigentümer kann sich dann nicht auf einen Bestandsschutz oder nachwirkenden Bestandsschutz berufen, wenn während einer Bewirtschaftungsbeschränkung im Sinne von $\$ 2 \mathrm{a}$ Abs. 1 ein besonders geschütztes Biotop entstanden ist, sofern die Wiederaufnahme der ursprünglichen Nutzung innerhalb von fünf, bei Waldflächen innerhalb von zehn Jahren nach Ende der Bewirtschaftungsbeschränkung erfolgt oder auf technischen Anlagen der öffentlichen Wasserwirtschaft ein besonders geschütztes Biotop entstanden ist.

OVG Bautzen, Beschluss vom 9.6.2009- 1 B 289/09-

\section{Aus den Gründen:}

Die Beschwerde des Antragstellers gegen den Beschluss des Verwaltungsgerichts vom 4.3.2009 hat keinen Erfolg. ... Der Antragsteller hat keinen Anspruch auf die Gewährung vorläufigen Rechtsschutzes gegen die mit Bescheid des Antragsgegners vom 2.12.2008 erlassene und für sofort vollziehbar erklärte Anordnung, die zwei Gräben auf seinem Flurstück F1 ... der Gemarkung K ... ... mit dem ausgehobenen und noch vor Ort lagernden Material zu verfüllen.

Nach $\$ 80$ Abs. 5 Satz 1 VwGO kann das Gericht auf Antrag die aufschiebende Wirkung eines für sofort vollziehbar erklärten Bescheides wiederherstellen, wenn nach der im Rahmen der im Verfahren des vorläufigen Rechtsschutzes allein möglichen und gebotenen summarischen Prüfung bei der Abwägung der Interessen das Suspensivinteresse des Antragstellers das Vollzugsinteresse überwiegt.

Das Verwaltungsgericht hat festgestellt, dass das Suspensivinteresse des Antragstellers das Interesse am sofortigen Vollzug der Anordnung nicht überwiegt. Es bestünden keine ernsthaften Zweifel an der Rechtmäßigkeit des Bescheides, dessen Vollziehung auch keine unbillige Härte darstelle. Die Verfügung beruhe auf $\int 41$ Abs. 1 des Sächsischen Gesetzes über Naturschutz und Landschaftspflege (Sächsisches Naturschutzgesetz - SächsNatSchG). Die Vorschrift ermächtige die Naturschutzbehörden zum Erlass erforderlicher Anordnungen zum Schutz von Natur und Landschaft und zur Einhaltung von Rechtsvorschriften. Auf dem Flurstück des Antragstellers befinde sich ein Biotop in Form einer seggen-und binsenreichen Nass- wiese. Hier seien sämtliche Handlungen verboten, die zu einer Zerstörung oder zu sonstigen erheblichen oder nachhaltigen Beeinträchtigungen führen würden. Die Entwässerung der geschützten Feuchtfläche durch die vom Antragsteller gehobenen Gräben stelle wohl einen solchen Eingriff dar. Umstände, die das Vorgehen des Antragstellers ausnahmsweise rechtfertigen könnten, lägen nicht vor. Das Biotop sei nicht erst infolge vertraglich vereinbarter Bewirtschaftungsbeschränkungen entstanden und seine Zerstörung nicht durch eine Wiederaufnahme der Bewirtschaftung innerhalb von 5 Jahren nach Aufgabe der ursprünglichen Nutzung erfolgt. Auch auf einen Bestandsschutz oder nachwirkenden Bestandsschutz könne der Antragsteller sich nicht berufen. Zum Zeitpunkt des Inkrafttretens des Sächsischen Naturschutzgesetzes seien Entwässerungsgräben auf dem Flurstück des Antragstellers jedenfalls nicht vorhanden gewesen. Auf dem Flurstück habe es wohl ursprünglich einen Graben gegeben, dieser sei aber mangels Instandhaltung und der Nutzung der Wiese als Weide zerstört und renaturiert gewesen. Es sei auch zweifelhaft, ob die Wiesenfläche überhaupt stillgelegt worden sei. Auch die besondere Bedeutung der Landwirtschaft für das öffentliche Wohl rechtfertigten die drohende Zerstörung des Biotops nicht. Aus sonstigen Fachgesetzen könne der Antragsteller ebenfalls keinen Anspruch auf die Gewährung von Ausnahmen vom Biotopschutz für sich herleiten. Der Antragsgegner habe das ihm in $\$ 41$ SächsNatSchG eingeräumte Ermessen erkannt und beanstandungsfrei ausgeübt.

Der Einwand des Antragstellers, er habe abweichend von der vom Verwaltungsgericht geäußerten Vermutung nachweislich etwa 2,3 ha seiner Bruchwiese ursprünglich als Weide genutzt und anschließend über mehrere Jahre hinweg stillgelegt, rechtfertigt keine Änderung des verwaltungsgerichtlichen Beschlusses. Die Entscheidung des Verwaltungsgerichts begründet sich in der Feststellung, dass das Biotop bereits vor einer - unterstellt werden könnenden - vorübergehenden Nutzungsbeschränkung entstanden war. Damit scheidet die Anwendung der Ausnahmeregelung in $\$ 26$ Abs. 4 Satz 3 SächsNatSchG von vornherein aus. Nach dieser Vorschrift gilt das in $\$ 26$ Abs. 2 SächsNatSchG normierte Verbot von Handlungen, die zu einer Zerstörung oder $\mathrm{zu}$ sonstigen erheblichen oder nachhaltigen Beeinträchtigungen der besonders geschützten Biotope führen können, ausnahmsweise und nur dann nicht, wenn das Biotop während der Bewirtschaftungsbeschränkung entstanden und die Wiederaufnahme der ursprünglichen Nutzung innerhalb von fünf Jahren nach Ende der Bewirtschaftungsbeschränkung erfolgt ist.

Auch die Ausführungen des Antragstellers zur Erforderlichkeit der Schutzmaßnahme rechtfertigen keine Änderung der Entscheidung des Verwaltungsgerichts. Über die Darstellung der im Gesetz festgeschriebenen Regelungen und in der Rechtsprechung hierzu entwickelten Grundsätze hinaus lassen sie nicht erkennen, welche Erwägungen zur Schutzwürdigkeit und -bedürftigkeit der Antragsgegner und das Verwaltungsgericht in ihre Entscheidung einzubeziehen gehabt hätten, aber nicht einbezogen haben. Antragsgegner und Gericht haben festgestellt, dass es sich bei der Nasswiese auf dem Flurstück des Antragstellers um ein Biotop i.S. v. $\$ 26$ SächNatSchG handelt. Hierin liegt seine grundsätzliche Schutzwürdigkeit begründet. Warum hiervon ausnahmsweise abzuweichen gewesen wäre, begründet der Antragsteller nicht und ist auch für den Senat nicht erkennbar. Das Verwaltungsgericht hat in seinem Beschluss ausgeführt, dass die vom Antragsteller gezogenen Gräben wegen ihrer Eignung, die Feuchtwiese tatsächlich zu entwässern, eine Gefahr für das Biotop darstellen. Welches mildere Mittel als die Verfüllung der Gräben zu veranlassen gewesen wäre, zeigt der Antragsteller nicht auf. 